\title{
Relación entre estilos parentales disfuncionales, empatía y variables sociodemográficas en estudiantes de Enfermería, Medicina Humana y Psicología
}

\author{
María Luisa Matalinares-Calvet; Ana Gloria Díaz-Acosta; Luisa Hortensia Rivas-Díaz; Carlos Alberto Arenas-Iparraguirre; \\ Deyvi Baca-Romero; Ornella Raymundo-Villalva; Nikolai Rodas-Vera
}

How to cite this article:

Matalinares-Calvet, M.L., Díaz-Acosta, A.G., Rivas-Díaz, L.H., Arenas-Iparraguirre, C.A., Baca-Romero, D., Raymundo-Villalva, O. \& Rodas-Vera, N. (2019). Dysfunctional parenting styles, empathy and socio-demographic variables in Nursing, Human Medicine and Psychology students. Acta Colombiana de Psicología, 22(2), 99-111. doi: http://www.doi.org/10.14718/ACP.2019.22.2.6

Recibido, junio 17/2018; Concepto de evaluación, julio 24/2018; Aceptado, noviembre 30/2018

\author{
María Luisa Matalinares-Calvet* \\ Universidad Nacional Mayor de San Marcos, Perú \\ ORCID: https://orcid.org/0000-0003-1052-6922 \\ Ana Gloria Díaz-Acosta \\ Universidad Nacional Mayor de San Marcos, Perú \\ ORCID: https://orcid.org/0000-0003-2720-0195 \\ Luisa Hortensia Rivas-Díaz \\ Universidad Nacional Mayor de San Marcos, Perú \\ ORCID: https://orcid.org/0000-0003-3540-8156 \\ Carlos Alberto Arenas-Iparraguirre \\ Universidad Nacional Mayor de San Marcos, Perú \\ ORCID: http://orcid.org/0000-0002-8342-2422 \\ Deyvi Baca-Romero \\ Universidad Inca Garcilaso de la Vega, Perú \\ ORCID: http://orcid.org/0000-0003-0184-0303 \\ Ornella Raymundo-Villalva \\ Universidad Científica del Sur, Perú \\ ORCID: https://orcid.org/0000-0001-8000-1422 \\ Nikolai Rodas-Vera \\ Universidad Cesar Vallejo, Perú \\ ORCID: https://orcid.org/0000-0001-6740-3099
}

\begin{abstract}
Resumen
El presente estudio tuvo como objetivo establecer la relación entre los estilos parentales disfuncionales y la empatía en estudiantes universitarios de Lima, además de comparaciones según su carrera profesional (Enfermería, Medicina Humana y Psicología). La muestra estuvo compuesta por 599 estudiantes de ambos sexos, con edades entre los 21 y 25 años, a quienes se les aplicó la Escala de Estilos Parentales Disfuncionales - de Parker et al. (1979)-, la Escala de Empatía Personal y Profesional -diseñada por Yaraskavitch et al. (2009)-, ambas adaptadas para los fines del estudio, y una ficha sociodemográfica elaborada por los autores. Los resultados muestran una relación no significativa entre los puntajes totales de estilos parentales disfuncionales y de empatía, aunque se obtuvo una relación inversa entre la subescala de estilo disfuncional paterno "autoritario-controlador" con las dimensiones "empatía personal cognitiva" y "empatía profesional cognitiva", y una relación directa entre la subescala de estilo disfuncional materno con las dimensiones "empatía personal emotiva" y "empatía profesional emotiva"; además de que, respecto al género, las mujeres presentaron mayor nivel de empatía, y, según carrera, los estudiantes de Enfermería presentaron mayores niveles de empatía, mientras que los de Medicina mostraron más estilos parentales disfuncionales. Finalmente, los estudiantes que llevaron a cabo cursos de desarrollo personal en su formación profesional presentaron un mayor nivel de empatía.

Palabras clave: empatía, estilos parentales disfuncionales, ciencias de la salud.
\end{abstract}

\footnotetext{
Facultad de Psicología, Universidad Nacional Mayor de San Marcos. Calle German Amezaga, 375, Lima. Tel.: $01516197000-3217$. maria_luisa93@hotmail.com
} 


\title{
Dysfunctional parenting styles, empathy and socio-demographic variables in Nursing, Human Medicine and Psychology students
}

\begin{abstract}
The objective of this study was to establish the relationship between dysfunctional parenting styles and empathy in Nursing, Human Medicine and Psychology students. The study's sample consisted of 599 students, from both genders, and from different cycles of their university studies. Their ages ranged between 21 and 25 years. To assess them, the Dysfunctional Parenting Styles Scale by Parker, et al. (1979) was used, previously adapted to Peruvian population. The participants were also evaluated with the Personal and Professional Empathy Scale designed by Yaraskavitch (2009), and completed a socio-demographic data sheet created ad hoc. General results showed no significant relationships between the dysfunctional parenting styles and empathy total scores. In contrast, considering the father figure, an inverse relationship between the authoritarian-controlling dysfunctional parenting style and the personal and professional cognitive empathy was found, while the indifference - negligence dysfunctional parenting style was inversely associated with the emotive personal empathy. As to the mother figure, there is a significant and direct relationship between authoritarian-controlling dysfunctional parenting style and personal and professional emotive empathy. It was also found that regarding gender, women have higher levels of empathy. With respect to professional degree courses, Nursing students have a higher level of empathy than Medicine and Psychology students, while Medicine students have more dysfunctional parenting styles than the Nursing and Psychology groups. Finally, students that had taken personal development courses showed a higher level of empathy. Key words: empathy, dysfunctional parental styles, majors in health sciences.
\end{abstract}

\section{Relaçâo entre estilos parentais disfuncionais, empatia e variáveis sociodemográficas em estudantes de Enfermagem, Medicina Humana e Psicologia}

Resumo

\begin{abstract}
O presente estudo teve como objetivo estabelecer a relação entre os estilos parentais disfuncionais e a empatia em estudantes universitários de Lima, além de comparações de acordo com a carreira profissional (Enfermagem, Medicina Humana e Psicologia). A amostra foi composta por 599 estudantes de ambos os sexos, com idades entre 21 e 25 anos, a quem foi aplicada a Escala de Estilos Parentais Disfuncionais - de Parker et a. (1979)-, a Escala de Empatia Pessoal e Profissional -desenhada por Yaraskavitch et al. (2009)-, ambas adaptadas para os fins do estudo, e uma ficha sociodemográfica elaborada pelos autores. Os resultados mostram uma relação não significativa entre as pontuações totais de estilos parentais disfuncionais e de empatia, mas obteve-se uma relação inversa entre a subescala de estilo disfuncional paterno "autoritário-controlador" com as dimensões "empatia pessoal cognitiva" e "empatia profissional cognitiva", e uma relação direta entre a subescala de estilo disfuncional materno com as dimensões "empatia pessoal emotiva" e "empatia profissional emotiva". Além disso, com respeito ao gênero, as mulheres apresentaram maior nível de empatia e, de acordo com o curso, os estudantes de Enfermaria apresentaram maiores níveis de empatia, enquanto os de Medicina mostraram mais estilos parentais disfuncionais. Finalmente, os estudantes que fizeram cursos de desenvolvimento pessoal em sua formação profissional apresentaram maior nível de empatia.

Palavras-chave: empatia, estilos parentais disfuncionais, ciências da saúde.
\end{abstract}

\section{Introducción}

El sistema de salud en la comunidad cumple el rol de promover en los individuos calidad de vida y capacidad de disfrute mediante la prevención y tratamiento de los problemas de salud. De este modo, el profesional en el área de la salud forma parte de un equipo que brinda un servicio a seres humanos que no solo presentan determinada sintomatología, sino que forman parte de una sociedad y tienen la necesidad de sentirse escuchados y atendidos con respeto y dignidad. Frente a ello, es importante investigar si los sistemas de salud en el país responden a las necesidades básicas de los ciudadanos tanto en la resolución de problemas médicos como en la necesidad básica de contacto humano en la búsqueda de alivio.

En este sentido, Cuba, Jurado y Estrella (2011) identificaron que un $52 \%$ de los usuarios del sistema de salud en Perú se siente satisfecho con la resolución de problemas, y que solo el $47 \%$ se sintió satisfecho en un nivel medio con la atención recibida, por lo cual resulta pertinente evaluar lo que sucede con el otro $50 \%$ de usuarios, además de reflexionar respecto a si el personal de salud está capacitado para brindar una atención humanizada, que, si bien se orienta 
a la resolución del problema médico, también debe generar bienestar y satisfacción en el usuario.

De este modo, se entiende que el arte de curar valida el encuentro personal entre el médico y el paciente con enfermedad, en donde se toma la relación diagnósticaterapéutica como imprescindible para una práctica humana (Lain, 1964). Teniendo esto en cuenta, el presente estudio busca identificar las habilidades empáticas en los estudiantes de carreras de salud y su relación con los estilos parentales disfuncionales y las variables sociodemográficas con el fin de explicar la importancia del desarrollo de esta habilidad a la hora de brindar una atención digna y humana. Tal como lo refiere Lain (1964), la relación con el semejante necesitado de ayuda debe estar enmarcada en una relación no meramente objetivante-que considere al otro como un puro objeto-, sino en una que lo considere como persona -como un ser individual, de naturaleza racional-, y como participante activo en una relación interhumana de ayuda -la consulta médica-.

Sobre la variable empatia, la presente investigación toma la propuesta hecha por Roger (1959; citado por Yarasckavitch, Regerhr, Hodges \& Haas, 2009), en donde esta es definida como la percepción precisa de la perspectiva de otro, considerando sus significados emocionales sin perder el propio punto de vista.

La mayoría de los expertos coinciden en que la empatía es una construcción multidimensional compuesta por procesos emotivos (sentimiento reflexivo) y cognitivos (pensamiento imaginativo). De acuerdo con la definición socio-psicológica de Davis (1994), los procesos emotivos reflejan la reactividad emocional general de una persona, por lo que la empatía emotiva implica la conciencia intuitiva de los sentimientos de otra persona, sin unirse directamente en la experiencia emocional completa del paciente; mientras que los procesos cognitivos son descritos como como una habilidad para asumir el punto de vista mental de otra persona, lo cual implica alejarse de la perspectiva propia para considerar los pensamientos o actitudes de otra persona dadas las circunstancias individuales de la misma. En conjunto, estos procesos emotivos y cognitivos reflejan la voluntad general de una persona para suprimir sus propias emociones y pensamientos con el fin de sentir e imaginar lo que es estar "en el lugar de otra persona".

$\mathrm{Al}$ revisar la literatura científica se encuentran diversos estudios para evaluar la empatía en estudiantes y profesionales de la salud. Por ejemplo, Sánchez (2010) realizó un estudio para identificar las relaciones entre la empatía médica, la sensibilidad emocional y las características psicosocio-demográficas en estudiantes de Medicina y médicos especialistas docentes de Venezuela, donde encontró alta empatía y sensibilidad interpersonal positiva en ambos grupos, así como empatía alta en estudiantes con responsabilidad social, jóvenes, mujeres y solteros, mientras que los alumnos de sexto año que no demostraron responsabilidad social y cuyos padres eran obreros especializados obtuvieron puntajes bajos en la sensibilidad interpersonal. Asimismo, en un estudio desarrollado por Esquerda, Yuguero, Viñas y Pifarré (2015) se estudió la evolución de la empatía en estudiantes de Medicina en España, y los resultados evidenciaron más empatía en las personas de género femenino, en quienes han realizado voluntariados y en los que han tenido algún amigo enfermo; mientras que los hombres, así como los que tienen otros familiares médicos, presentaron menor empatía.

En Brasil, Moreto, Gonzáles, Pessini y Craice (2014) estudiaron la erosión de la empatía en estudiantes de Medicina, y concluyeron que el nivel de empatía puede cambiar y que los planes de estudio en las ciencias de la salud pueden influir en el desarrollo del nivel de empatía, por lo que resultaría conveniente enfocar las acciones educativas en mantener o incrementar la empatía al trabajar más que en enseñar cosas nuevas, en "enseñar a ser empático". De igual modo, en Colombia, Bilbao et al. (2015) realizaron un análisis sobre la orientación empática en estudiantes de Medicina de dos universidades e identificaron que el nivel de orientación empática cuenta con valores bajos, en general. Por otro lado, en Chile, Díaz, Salas, Bracho y Ocaranza (2015) estimaron los niveles de empatía en estudiantes de kinesiología y encontraron que presentaban valores relativamente altos de empatía. Pero, en Perú, Salcedo-Rioja y Díaz-Narváez (2015) analizaron la empatía en estudiantes de odontología de la Universidad Nacional Mayor de San Marcos, sin encontrar diferencias significativas en la empatía en cuanto al año de la carrera ni con respecto al género.

En general, diversos estudios evidencian la existencia de variables familiares, institucionales y sociodemográficas que inciden sobre el nivel de empatía en los estudiantes de carreras de la salud, lo que justificaría la importancia de su estudio. Al respecto, Madera, Tirado y González (2015), por ejemplo, estudiaron los factores relacionados con la empatía en estudiantes de Medicina de la Universidad de Cartagena y concluyeron que los niveles de empatía pueden variar dependiendo del sexo, la primera opción de estudio, el promedio, el rendimiento académico y la funcionalidad familiar, por lo cual resaltan la importancia de implementar estrategias pedagógicas en las áreas humanas que involucren a las familias de los educandos con el fin de mejorar los niveles de empatía y la atención en salud.

Por otra parte, en cuanto a los estilos parentales disfuncionales, existe una constelación de actitudes dañinas en la crianza de los niños que, en conjunto, crean un clima emocional disfuncional en el que se ponen de manifiesto los comportamientos de los padres en relación con sus hijos. Al respecto, Parker, Tupling y Brown (1979) describen los 
102

estilos parentales disfuncionales de indiferencia-negligencia y autoritario-controlador; dicotomía -también desarrollada por Baumrind (1996)- descrita como posturas extremas en donde ni la actitud conservadora y autoritaria ni la posición más liberal y permisiva ofrecen a los progenitores un modelo adecuado para la crianza de sus hijos.

Ahora bien, partiendo de los estudios realizados en 1964 por Laín, Camacho (2006) realizó una publicación sobre la relación médico-paciente donde recomienda que los profesionales sanitarios deben recibir una formación específica sobre la relación interpersonal y la manera de comunicarse con las personas que van a tratar; pero un estudio reciente, realizado por García y Gálvez (2016), señaló que solo siete de cada diez usuarios del Seguro Social de Salud de Perú y cinco de cada diez del Ministerio de Salud de Perú se encontraban satisfechos con la atención empática del personal de salud.

Teniendo en cuenta lo antes descrito, brindar una mejor atención por parte del personal de salud requiere revisar, en parte, la formación profesional que recibe; ejercicio que evidencia la importancia que tiene el hecho de que las universidades incluyan aspectos relacionados con la formación en valores y que enseñen, entre otros aspectos, a ser empáticos. Mantener dicha habilidad es una forma importante de humanizar la educación en las carreras de salud, ya que identificar los factores sociales y familiares de las personas que consultan ayudará a explicar mejor la enfermedad y sus futuras formas de intervención. Todo esto sin olvidar que el desarrollo de la empatía también deviene de los estilos de crianza, ya que se debe considerar al individuo como el resultado de sus experiencias vividas y significativas durante su desarrollo en la infancia.

En este contexto, el presente estudio busca establecer la relación entre los estilos parentales disfuncionales y la empatía en estudiantes de Lima y comparar sus resultados según su carrera profesional (Enfermería, Medicina Humana o Psicología).

\section{Método}

\section{Tipo de estudio}

En concordancia con el objetivo formulado, se empleó un tipo de investigación sustantiva-descriptiva, con un análisis descriptivo, comparativo y correlacional (Sánchez $\&$ Reyes, 2015). El diseño de investigación utilizado en el presente estudio fue no experimental, transeccional y correlacional (Hernández, Fernández \& Baptista, 2016).

\section{Participantes}

La población objetivo fue los estudiantes matriculados de tercer a noveno ciclo en las carreras de Enfermería,
Medicina y Psicología de una universidad nacional y privada de Lima, Perú. La muestra fue seleccionada por medio de un muestreo intencional (Otzen \& Monterola, 2017) y estuvo compuesta por 599 estudiantes con edades entre los 21 y los 25 años $(\mathrm{M}=23.20, \mathrm{DS}=3.34)$, con un mayor porcentaje de mujeres (65.9\%) que de hombres (34.1\%). En resumen, se recogió información de estudiantes desde el tercer año de formación en las tres carreras, de los cuales el 54.6\% cursaba el sexto año, y se obtuvo un porcentaje similar de estudiantes para cada una de las tres carreras: Enfermería (34.4\%), Medicina (34.6\%) y Psicología (31.1\%). El $32.1 \%$ del total de estudiantes señaló haber llevado un curso de desarrollo personal en su formación universitaria.

\section{Aspectos éticos}

La información recopilada de los participantes fue tratada de forma anónima, previo consentimiento informado, y la investigación fue evaluada como proyecto por el Vicerrectorado de Investigación de la Universidad Nacional Mayor de San Marcos.

\section{Instrumentos}

La técnica de estudio fue la encuesta y los instrumentos utilizados fueron la Escala de Estilos Parentales Disfuncionales (EEPD), de Parker et al. (1979); la Escala de Empatía Personal y Profesional (EEPP), de Yarasckavitch et al. (2009); y una ficha sociodemográfica elaborada por los autores del presente estudio.

Escala de Estilos Parentales Disfuncionales (EEPD). Es una prueba elaborada por Parker et al. (1979) con el fin de evaluar estilos parentales disfuncionales, que consta de 15 ítems, y que se administra de forma individual o colectiva en un tiempo promedio de 10 a 15 minutos. En el presente estudio, el análisis de confiabilidad por consistencia interna de las subescalas padre y madre alcanzó un nivel moderado, con valores de .88 y .89 , respectivamente; valores superiores a los reportados por Matalinares, Raymundo y Baca (2014), en donde se encontró un alfa de Cronbach de .82 para la escala padre y de .78 para la escala madre.

Se analizó la validez del instrumento mediante el análisis factorial exploratorio (AFE) de ambas subescalas, donde se encontró una distribución de los ítems en dos factores: la subescala padre, con un $43.79 \%$, y la subescala madre, con un $40.20 \%$ de varianza explicada. El Factor 1 de la subescala padre y el Factor 2 de la subescala madre recibieron la denominación de "indiferencia-negligencia" según el análisis de contenido de los ítems; mientras que el rótulo de "autoritario-controlador" se asignó al Factor 2 de la subescala padre y al Factor 1 de la subescala madre. Si bien estos 
resultados no coinciden con el estudio de Matalinares et al. (2014), donde se reportaron tres factores (subescala padre, con $53.82 \%$ de varianza explicada; subescala madre, con $49.26 \%$ de varianza explicada), esta incongruencia puede darse debido a que la muestra del presente estudio estuvo compuesta por estudiantes universitarios, mientras que la del estudio previo era de estudiantes de nivel secundario.

Los baremos fueron trabajados con puntajes directos, donde los puntos de corte para la subescala padre fueron de 14-17 para bajo, de 18-22 para medio y de 23-56 para alto; y para la subescala madre fueron de 14-17 para bajo, de 18-22 para medio y de 23-56 para alto.

Escala de Empatía Personal y Profesional (EEPP). Es una prueba elaborada por Yarasckavitch et al. (2009), traducida al español por Carrasco, Fasce y Pérez (2014) -mediante un proceso de retrotraducción-, conformada por 52 ítems con escala de respuesta tipo Likert de cinco puntos. Específicamente, 26 ítems evalúan la empatía profesional y los 26 restantes evalúan la empatía personal; siete ítems tanto de la escala personal como de la profesional (14 en total) son inversos: 3, 7, 8, 9, 15, 23 y 26.

La confiabilidad de esta prueba se analizó por medio del método de consistencia interna, pero no se encontraron estudios previos del EEPPen la literatura nacional. Como resultado, en el análisis preliminar de ambas subescalas los ítems inversos $(3,7,8,13,20,25$ y 29) obtuvieron valores de correlación ítem-test por debajo de .20, por lo que fueron eliminados, y un segundo análisis de confiabilidad determinó valores adecuados y un alfa de Cronbach de .80 para la escala de empatía personal y de .85 para la escala de empatía profesional.

Para la validez del instrumento se hizo uso del análisis factorial exploratorio (AFE) tanto para la escala empatía personal como para la escala empatía profesional, donde se encontraron dos factores predominantes: la empatía emotiva y la empatía cognitiva. La escala empatía personal quedo explicada en un $30.80 \%$ de la varianza por ambos factores $(\mathrm{KMO}=.849$; prueba de Bartlett con $p<.05)$, mientras que la escala empatía profesional lo hizo con un $44.16 \%$ de la varianza explicada $(\mathrm{KMO}=.889$; prueba de Bartlett con $p<.05)$. Estos dos factores siguieron la misma distribución de ítems que el estudio de Carrasco et al. (2014).

Los baremos utilizados para el EEPP fueron los puntajes directos, en donde la dimensión empatía personal con un puntaje de 17 a 44 correspondía a un nivel bajo, mientras que uno de 45 a 50 correspondía a un nivel medio, y un puntaje de 51 y 68, a un nivel alto. Asimismo, la dimensión de empatía profesional con una puntuación de 17 a 42 correspondía a un nivel bajo, mientras que una de 43 a 49 correspondía a un nivel medio, y una de 50 y 68, a un nivel alto.

\section{Ficha sociodemográfica}

Se realizó una encuesta estructurada para el llenado individual por parte de cada estudiante, donde se consignaba la información relacionada con los datos familiares, la elección de carrera, las motivaciones por las que estudia su carrera, los datos de la enseñanza universitaria, y si llevaron o no a cabo cursos de desarrollo personal en su formación universitaria.

\section{Procedimiento de recolección de datos}

Los instrumentos se aplicaron en los ambientes de las universidades tras la solicitud de permiso al docente responsable de aula. Dicha aplicación estuvo a cargo de profesionales de la salud (psicólogos) debidamente capacitados para ello, siguiendo siempre el mismo orden: en primer lugar, los estudiantes eran informados de los aspectos éticos del estudio; y luego, tras la firma del consentimiento explicito (consentimiento informado), se procedía con la aplicación de la ficha sociodemográfica, seguida de la Escala de Empatía y la Escala de Estilos Parentales Disfuncionales.

\section{Análisis de la información}

Los datos recuperados fueron transcritos a una plantilla de base de datos posteriormente exportada al software SPSS, versión 21. Después de esto, se utilizaron estadísticos de frecuencia y porcentaje para el análisis descriptivo, y para un mejor análisis de las variables de estilos parentales y empatía se agruparon las categorías baja, media y alta. Seguidamente, los análisis inferenciales - procesados con los puntajes directos de los instrumentos-fueron realizados con pruebas estadísticas de tipo no paramétricas, debido a la ausencia de un muestreo probabilístico que garantizara la representatividad de la muestra (Manterola \& Otzen, 2017).

\section{Resultados}

A continuación se presenta la información descriptiva de las variables de estudio (estilos parentales disfuncionales y empatía) a través de los niveles bajo, medio y alto, en relación con la variable sociodemográfica carrera profesional (Enfermería, Medicina y Psicología). Solo se utilizó dicha variable sociodemográfica debido a que existía un porcentaje similar de estudiantes en las tres carreras (alrededor del $30 \%$ ). Posteriormente, se presenta el análisis inferencial a partir del establecimiento de correlaciones y comparaciones acorde a los objetivos del estudio, por medio de los puntajes directos de las variables.

\section{Resultados descriptivos}

En la Tabla 1 se presentan los resultados de 572 estudiantes universitarios, debido a que 27 de ellos no percibieron 
Tabla 1.

Niveles de la subescala padre del EEPD y sus dimensiones según la carrera profesional de estudiantes universitarios $(N=572)$

\begin{tabular}{ccccccc}
\hline & \multicolumn{5}{c}{ Carrera profesional } & \multicolumn{2}{c}{ Psicología } \\
Subescala y dimensiones según niveles & \multicolumn{2}{c}{$\begin{array}{c}\text { Enfermería } \\
(\mathrm{n}=194)\end{array}$} & \multicolumn{2}{c}{$\begin{array}{c}\text { Medicina } \\
(\mathrm{n}=198)\end{array}$} \\
\cline { 2 - 7 } & $\mathrm{N}$ & $\%$ & $\mathrm{~N}$ & $\%$ & $\mathrm{~N}$ & $\%$ \\
\cline { 2 - 7 } & & & & & & \\
Subescala padre & 42 & 7.3 & 58 & 10.12 & 43 & 7.5 \\
Alta & 85 & 14.9 & 86 & 15.0 & 94 & 16.5 \\
Media & 67 & 11.78 & 54 & 9.4 & 43 & 7.5 \\
Baja & & & & & & \\
Alta & 43 & 7.5 & 50 & 8.7 & 31 & 5.4 \\
Media & 109 & 19.1 & 121 & 21.2 & 100 & 17.5 \\
Baja & 42 & 7.3 & 27 & 4.7 & 49 & 8.6 \\
\hline Altaferencia-negligencia & & & & & & \\
Media & 25 & 4.4 & 46 & 8.0 & 45 & 7.9 \\
Baja & 118 & 20.6 & 102 & 17.8 & 95 & 16.6 \\
\hline Total & 51 & 8.9 & 50 & 8.87 & 40 & 7.1 \\
\hline Autoritario-controlador & 194 & 100.0 & 198 & 100.0 & 180 & 100.0 \\
\hline
\end{tabular}

Nota. $\mathrm{n}=$ número de casos.

la presencia de una figura paterna en sus primeros 16 años de vida. Así, la muestra de los estilos parentales disfuncionales en la subescala padre puntuó predominantemente en un nivel medio en todas las carreras, sin embargo, hubo un porcentaje ligeramente mayor de estudiantes de Medicina en el nivel alto de estilo disfuncional paterno, al igual que en lo observado en las dimensiones autoritario-controlador e indiferencia-negligencia.
En la Tabla 2 se pueden observar los resultados de 598 estudiantes universitarios con respecto a la presencia de la figura materna -un estudiante no percibió en sus primeros 16 años de vida esta figura-. Los estilos parentales disfuncionales de la subescala madre alcanzaron un nivel medio en todas las carreras profesionales, aunque se encontró un porcentaje ligeramente mayor de estudiantes de Medicina en el nivel alto de estilo disfuncional materno. Esto mismo

Tabla 2.

Niveles de la subescala madre del EEPD y sus dimensiones según la carrera profesional de estudiantes universitarios $(N=598)$

\begin{tabular}{ccccccc}
\hline & \multicolumn{9}{c}{ Carrera profesional } \\
\cline { 2 - 7 } Subescala y dimensiones según niveles & \multicolumn{2}{c}{$\begin{array}{c}\text { Enfermería } \\
(\mathrm{n}=205)\end{array}$} & \multicolumn{2}{c}{$\begin{array}{c}\text { Medicina } \\
(\mathrm{n}=207)\end{array}$} & \multicolumn{2}{c}{$\begin{array}{c}\text { Psicología } \\
(\mathrm{n}=186)\end{array}$} \\
\cline { 2 - 7 } & $\mathrm{N}$ & $\%$ & $\mathrm{~N}$ & $\%$ & $\mathrm{~N}$ & $\%$ \\
\hline Subescala madre & 36 & 6.0 & 48 & 8.0 & 38 & 6.4 \\
Alta & 130 & 21.7 & 113 & 18.9 & 114 & 19.1 \\
Media & 39 & 6.5 & 46 & 7.7 & 34 & 5.7 \\
Baja & & & & & & \\
Alta & 46 & 7.7 & 49 & 8.2 & 52 & 8.7 \\
Media & 109 & 18.2 & 113 & 18.9 & 72 & 12.0 \\
Baja & 50 & 8.4 & 45 & 7.5 & 62 & 10.4 \\
\hline Indiferencia-negligencia & & & & & & \\
Alta & 46 & 7.7 & 57 & 9.5 & 42 & 7.0 \\
Media & 120 & 20.1 & 102 & 17.1 & 112 & 18.7 \\
Baja & 39 & 6.5 & 48 & 8.0 & 32 & 5.4 \\
\hline Total & 205 & 100.0 & 207 & 100.0 & 186 & 100.0 \\
\hline Autoritario-controlador & & & &
\end{tabular}

Nota. $\mathrm{n}=$ número de casos. 
Tabla 3.

Niveles de empatía personal del EEPD y sus dimensiones según la carrera profesional de estudiantes universitarios $(N=599)$

\begin{tabular}{ccccccc}
\hline & \multicolumn{9}{c}{ Carrera profesional } \\
\cline { 2 - 7 } Variable y dimensiones según niveles & \multicolumn{2}{c}{$\begin{array}{c}\text { Enfermería } \\
(\mathrm{n}=206)\end{array}$} & $\begin{array}{c}\text { Medicina } \\
(\mathrm{n}=207)\end{array}$ & \multicolumn{2}{c}{$\begin{array}{c}\text { Psicología } \\
(\mathrm{n}=186)\end{array}$} \\
\cline { 2 - 7 } & $\mathrm{N}$ & $\%$ & $\mathrm{~N}$ & $\%$ & $\mathrm{~N}$ & $\%$ \\
\hline Empatía personal & & & & & & \\
Alta & 68 & 11.4 & 46 & 7.7 & 13 & 2.2 \\
Media & 112 & 18.7 & 114 & 19.0 & 102 & 17.0 \\
Baja & 26 & 4.3 & 47 & 7.9 & 71 & 11.8 \\
\hline Alta & & & & & & \\
Media & 60 & 10.0 & 41 & 6.8 & 12 & 2.0 \\
Baja & 107 & 17.9 & 111 & 18.5 & 106 & 17.7 \\
Empatía personal emotiva & 39 & 6.5 & 55 & 9.2 & 68 & 11.4 \\
\hline Alta & & & & & & \\
Media & 45 & 7.5 & 39 & 6.5 & 24 & 4.0 \\
Baja & 131 & 21.9 & 121 & 20.2 & 103 & 17.2 \\
Total & 30 & 5.0 & 47 & 7.9 & 59 & 9.8 \\
\hline Empatía personal cognitiva & 206 & 100.0 & 207 & 100.0 & 186 & 100.0 \\
\hline
\end{tabular}

Nota. $\mathrm{n}=$ número de casos.

se ve reflejado en las dimensiones autoritario-controlador e indiferencia-negligencia.

Por otra parte, de acuerdo con lo presentado en la Tabla 3, se encontró un nivel medio de empatía personal en todas las carreras profesionales, aunque hubo un mayor porcentaje de estudiantes de Enfermería con un nivel alto de empatía personal, y un mayor porcentaje de estudiantes de Psicología con un nivel bajo en esta variable. Adicionalmente, se puede apreciar una situación similar en las dos dimensiones: empatía personal emotiva y empatía personal cognitiva.

Por otra parte, la empatía profesional alcanzó un nivel medio en los estudiantes de todas las carreras profesionales (véase Tabla 4), aunque se puede observar un porcentaje ligeramente mayor en los estudiantes de Enfermería, con un nivel alto de empatía profesional y de sus dimensiones en comparación con los estudiantes de Psicología, quienes

Tabla 4.

Niveles de empatía profesional del EEPD y sus dimensiones según la carrera profesional de estudiantes universitarios $(N=599)$

\begin{tabular}{ccccccc}
\hline & \multicolumn{9}{c}{ Carrera profesional } \\
\cline { 2 - 7 } Escala y dimensiones según niveles & \multicolumn{2}{c}{$\begin{array}{c}\text { Enfermería } \\
(\mathrm{n}=206)\end{array}$} & $\begin{array}{c}\text { Medicina } \\
(\mathrm{n}=207)\end{array}$ & \multicolumn{2}{c}{$\begin{array}{c}\text { Psicología } \\
(\mathrm{n}=186)\end{array}$} \\
\cline { 2 - 7 } & $\mathrm{N}$ & $\%$ & $\mathrm{~N}$ & $\%$ & $\mathrm{~N}$ & $\%$ \\
\hline Empatía profesional & & & & & & \\
Alta & 78 & 13.0 & 48 & 8.0 & 12 & 2.0 \\
Media & 104 & 17.4 & 111 & 18.5 & 92 & 15.4 \\
Baja & 24 & 4.0 & 48 & 8.0 & 82 & 13.7 \\
\hline Alta & & & & & & \\
Media & 58 & 9.7 & 41 & 6.8 & 11 & 1.8 \\
Baja & 120 & 20.0 & 118 & 19.7 & 118 & 19.7 \\
\hline Alta & 28 & 4.7 & 48 & 8.0 & 57 & 9.6 \\
Media & & & & & & \\
Baja & 69 & 11.5 & 41 & 6.8 & 22 & 3.8 \\
Total & 120 & 20.0 & 133 & 22.2 & 96 & 16.0 \\
\hline Empatía profesional cognitiva & 17 & 2.8 & 33 & 5.5 & 68 & 11.4 \\
\hline
\end{tabular}

Nota. $\mathrm{n}=$ número de casos. 
tienen un porcentaje ligeramente mayor en el nivel bajo de empatía profesional y sus dimensiones.

\section{Resultados inferenciales}

Para el análisis inferencial, se trabajó con los puntajes directos de las variables de estudio y sus dimensiones, $\mathrm{y}$, ante la ausencia de un muestreo probabilístico, se utilizó la prueba no paramétrica rho de Spearman para el análisis correlacional, y las pruebas no paramétricas U de MannWhitney y Kruskal-Wallis para el análisis comparativo.

En la Tabla 5 se pueden observar las correlaciones inversas y de magnitud pequeña entre las dimensiones empatía personal cognitiva y empatía profesional cognitiva con la dimensión autoritario-controlador, lo cual sugiere la ausencia de una correlación, dado el tamaño de muestra -grande $(\mathrm{N}=572)-$, al igual que entre la dimensión personal emotiva y el estilo disfuncional paterno con la indiferencia-negligencia. El tamaño de efecto estimado en las correlaciones significativas fue pequeño (Castillo, 2014). Teniendo esto en cuenta, se requiere que en futuras investigaciones se profundice en el análisis de dichas variables.

Por otra parte, de acuerdo con la Tabla 6, se encontraron asociaciones directas de las dimensiones empatía personal emotiva y empatía profesional emotiva con los estilos disfuncionales maternos de autoritario-controlador. Es decir,

Tabla 5.

Correlaciones entre los estilos parentales disfuncionales paternos y la empatía personal y profesional de estudiantes universitarios $(N=572)$

\begin{tabular}{|c|c|c|c|c|}
\hline \multirow{2}{*}{ Variable y dimensiones } & & \multicolumn{3}{|c|}{ Subescala padre } \\
\hline & & Indiferencia-negligencia & Autoritario-controlador & Subescala total \\
\hline \multirow{2}{*}{ Empatía personal } & Rho & .052 & -.029 & .003 \\
\hline & $r^{2}$ & - & - & - \\
\hline \multirow{2}{*}{ Empatía personal emotiva } & Rho & $.095 *$ & .037 & .055 \\
\hline & $r^{2}$ & .009 & - & - \\
\hline \multirow{2}{*}{ Empatía personal cognitiva } & Rho & -.044 & $-.117 * *$ & $-.085^{*}$ \\
\hline & $r^{2}$ & - & .014 & .007 \\
\hline \multirow{2}{*}{ Empatía profesional } & Rho & .009 & -.047 & -.028 \\
\hline & $r^{2}$ & - & - & - \\
\hline \multirow{2}{*}{ Empatía profesional emotiva } & Rho & .048 & .039 & .044 \\
\hline & $r^{2}$ & - & - & - \\
\hline \multirow{2}{*}{ Empatía profesional cognitiva } & Rho & -.054 & $-.159 * *$ & $-.126 * *$ \\
\hline & $r^{2}$ & - & .025 & .016 \\
\hline
\end{tabular}

Nota. $* * p<.01 ; * p<.05 ; r^{2}=$ coeficiente de determinación.

Tabla 6.

Correlaciones entre los estilos parentales disfuncionales maternos y la empatía personal y profesional en estudiantes universitarios $(N=598)$

\begin{tabular}{|c|c|c|c|c|}
\hline \multirow{2}{*}{ Variable y dimensiones } & & \multicolumn{3}{|c|}{ Subescala madre } \\
\hline & & Indiferencia-negligencia & Autoritario-controlador & Subescala total \\
\hline \multirow{2}{*}{ Empatía personal } & Rho & .049 & .097 & $.098^{*}$ \\
\hline & $r^{2}$ & - & - & .010 \\
\hline \multirow{2}{*}{ Empatía personal emotiva } & Rho & .075 & $.107 * *$ & $.116^{* *}$ \\
\hline & $r^{2}$ & - & .012 & .014 \\
\hline \multirow{2}{*}{ Empatía personal cognitiva } & Rho & -.014 & .015 & .005 \\
\hline & $r^{2}$ & - & - & - \\
\hline \multirow{2}{*}{ Empatía profesional } & Rho & .034 & .071 & .066 \\
\hline & $r^{2}$ & - & - & \\
\hline \multirow{2}{*}{ Empatía profesional emotiva } & Rho & .037 & $.093 *$ & $.084 *$ \\
\hline & $r^{2}$ & - & .009 & .007 \\
\hline \multirow{2}{*}{ Empatía profesional cognitiva } & Rho & .010 & .004 & .002 \\
\hline & $r^{2}$ & - & - & - \\
\hline
\end{tabular}

Nota: $* * p<.01, * p<.05, r^{2}=$ coeficiente de determinación. 
que una empatía personal emotiva mayor estaría asociada a un mayor estilo disfuncional materno de autoritario-controlador, al igual que con la dimensión empatía profesional emotiva. Cabe destacar que el tamaño de efecto $\left(r^{2}\right)$ en las correlaciones fue pequeño (Castillo, 2014).

Adicionalmente, las diferencias según el sexo, estimadas por medio de la prueba U de Mann-Whitney, fueron significativas en la variable empatía personal y su dimensión emotiva; en ambas hubo un mejor nivel de empatía a favor de las mujeres. De forma similar, se encontraron diferencias significativas a favor de las mujeres en la variable empatía profesional y sus dimensiones emotivas y cognitiva (véase Tabla 7). En todas las diferencias encontradas se observó un tamaño de efecto (PS) de nivel medio (Castillo, 2014). Es importante mencionar que no se encontraron diferencias según el género en los estilos parentales disfuncionales.

Asimismo, en la Tabla 8 se pueden observar las diferencias según edad en las variables de estudio, estimadas por medio la prueba de Kruskal-Wallis, donde la variable empatía personal presentó diferencias a favor de los estudiantes con menos de 21 años, y la dimensión empatía personal emotiva lo hizo a favor de los estudiantes de 22 años. De igual forma,

Tabla 7.

Comparación de los estilos parentales disfuncionales y empatía personal y profesional, según el género, en estudiantes universitarios $(N=599)$

\begin{tabular}{lcccc}
\hline \multirow{2}{*}{ Variables y dimensiones } & \multicolumn{2}{c}{ Rango promedio } & U de Mann- \\
\cline { 2 - 4 } & Hombres & $\begin{array}{c}\text { Mujeres } \\
(\mathrm{n}=395)\end{array}$ & PS \\
\hline Estilo disfuncional paterno & 296.80 & 281.01 & 35064.00 & - \\
Indiferencia-negligencia & 299.09 & 279.78 & 34608.50 & - \\
Autoritario-controlador & 293.52 & 282.75 & 35716.50 & - \\
\hline Estilo disfuncional materno & 297.16 & 300.71 & 39710.50 & - \\
Indiferencia-negligencia & 302.02 & 298.19 & 39673.00 & - \\
Autoritario-controlador & 295.48 & 301.58 & 39367.00 & .41 \\
\hline Empatía personal & 266.44 & 317.33 & $33444.500^{* *}$ & .49 \\
Emotiva & 263.20 & 319.00 & $32783.500^{* *}$ & .42 \\
Cognitiva & 294.74 & 302.72 & 39217.000 & .42 \\
\hline Empatía profesional & 262.22 & 319.51 & $32583.00 * *$ & .44 \\
Emotiva & 268.06 & 316.49 & $33775.00 * *$ & .40 \\
Cognitiva & 275.89 & 312.45 & $35371.50 *$ & 40 \\
\hline
\end{tabular}

Nota. $* * p<.01 ; * p<.05 ; \mathrm{PS}=$ probabilidad de superioridad.

Tabla 8.

Comparación de los estilos parentales disfuncionales y la empatía personal y profesional, según la edad, en estudiantes universitarios $(N=599)$

\begin{tabular}{|c|c|c|c|c|c|c|c|c|c|}
\hline \multirow[b]{2}{*}{ Variable y dimensiones } & \multicolumn{7}{|c|}{ Rango promedio según la edad (años) } & \multirow[b]{2}{*}{$\begin{array}{l}\text { Chi-cuadrado } \\
\text { (gl) }\end{array}$} & \multirow[b]{2}{*}{$\begin{array}{c}\text { Vde } \\
\text { Cramer }\end{array}$} \\
\hline & $\begin{array}{c}\text { Menos } \\
\text { de } 21\end{array}$ & 21 & 22 & 23 & 24 & 25 & $\begin{array}{c}\text { Más de } \\
25\end{array}$ & & \\
\hline Estilo disfuncional paterno & 281.5 & 284.1 & 272.5 & 308.2 & 277.2 & 303.0 & 280.8 & $3.353(6)$ & - \\
\hline Indiferencia-negligencia & 300.0 & 295.3 & 287.8 & 302.8 & 229.8 & 304.2 & 277.4 & $10.649(6)$ & - \\
\hline Autoritario-controlador & 266.1 & 272.0 & 270.1 & 310.9 & 304.0 & 297.9 & 288.7 & $6.051(6)$ & - \\
\hline Estilo disfuncional materno & 305.6 & 290.3 & 292.7 & 313.1 & 278.7 & 302.5 & 306.1 & $2.230(6)$ & - \\
\hline Indiferencia-negligencia & 323.1 & 282.9 & 309.3 & 296.7 & 256.5 & 305.0 & 308.9 & $7.593(6)$ & - \\
\hline Autoritario-controlador & 293.4 & 293.7 & 289.9 & 313.0 & 296.6 & 302.7 & 305.7 & $1.301(6)$ & - \\
\hline Empatia personal & 355.8 & 334.3 & 291.4 & 325.8 & 219.7 & 265.5 & 275.9 & $33.816(6)^{* *}$ & .10 \\
\hline Emotiva & 347.8 & 350.4 & 303.9 & 320.4 & 234.1 & 266.7 & 253.6 & $34.459(6)^{* *}$ & .10 \\
\hline Cognitiva & 333.7 & 304.0 & 287.2 & 311.0 & 243.4 & 275.7 & 318.5 & $13.970(6)^{*}$ & .06 \\
\hline Empatía profesional & 347.7 & 336.5 & 302.9 & 312.3 & 249.0 & 297.6 & 245.4 & $26.929(6)^{* *}$ & .09 \\
\hline Emotiva & 337.5 & 346.7 & 326.6 & 312.3 & 265.7 & 279.2 & 223.8 & $36.134(6) * *$ & .10 \\
\hline Cognitiva & 329.5 & 310.4 & 274.3 & 304.1 & 250.4 & 311.2 & 310.6 & $11.384(6)$ & - \\
\hline
\end{tabular}

Nota. $g l=$ grados de libertad; $* p<.05 ; * * p<.01$. 
se encontraron diferencias en la variable empatía profesional a favor de los estudiantes de menos de 21 años y en la dimensión empatía profesional emotiva a favor de los estudiantes de 22 años. El tamaño de efecto, $V$ de Cramer, fue de nivel pequeño en las diferencias significativas (Castillo, 2014), lo que sugiere revisar resultados en futuras investigaciones. Cabe señalar que no se obtuvieron diferencias significativas en los estilos disfuncionales parentales.

Además de lo anterior, se utilizó la prueba de KruskalWallis para las comparaciones según la carrera profesional (véase Tabla 9), a partir de la cual se encontraron diferencias significativas en las dimensiones autoritario-controlador e indiferencia-negligencia en el estilo disfuncional paterno, dado que los estudiantes de Medicina obtuvieron un mayor puntaje, aunque con un tamaño de efecto pequeño (Castillo, 2014). Igualmente, se encontraron diferencias significativas de mayor empatía personal y profesional en estudiantes de Enfermería, con un tamaño de efecto mediano (Castillo, 2014).

Por último, se realizaron comparaciones a partir de si los estudiantes habían llevado a cabo o no cursos de desarrollo personal en la carrera profesional. En la Tabla 10 se presentan las diferencias significativas identificadas en la dimensión autoritario-controlador de la subescala padre, con un mayor puntaje en los estudiantes que refirieron no llevar cursos de desarrollo personal. Las variables empatía personal y profesional, y sus dimensiones, también lograron diferencias

Tabla 9.

Comparación de los estilos parentales disfuncionales y la empatía personal y profesional, según la carrera profesional, en estudiantes universitarios $(N=599)$

\begin{tabular}{|c|c|c|c|c|c|}
\hline \multirow[b]{2}{*}{ Variable y dimensiones } & \multicolumn{3}{|c|}{ Rango promedio según carrera profesional } & \multirow[b]{2}{*}{$\begin{array}{l}\text { Chi-cuadrado } \\
\text { (gl) }\end{array}$} & \multirow[b]{2}{*}{ Vde Cramer } \\
\hline & $\begin{array}{c}\text { Enfermería } \\
(\mathrm{n}=206)\end{array}$ & $\begin{array}{l}\text { Medicina } \\
(\mathrm{n}=207)\end{array}$ & $\begin{array}{l}\text { Psicología } \\
(\mathrm{n}=186)\end{array}$ & & \\
\hline Estilo disfuncional paterno & 266.1 & 302.8 & 290.6 & $5.010(2)$ & - \\
\hline Indiferencia-negligencia & 283.4 & 310.1 & 263.9 & $7.698(2)^{*}$ & .08 \\
\hline Autoritario-controlador & 259.9 & 293.0 & 308.0 & $8.501(2)^{*}$ & .09 \\
\hline Estilo disfuncional materno & 300.0 & 305.7 & 292.0 & $.629(2)$ & - \\
\hline Indiferencia-negligencia & 299.8 & 308.4 & 289.2 & $1.278(2)$ & - \\
\hline Autoritario-controlador & 300.1 & 303.8 & 294.1 & $.317(2)$ & - \\
\hline Empatía personal & 366.0 & 308.4 & 217.5 & $72.921(2)^{* *}$ & .25 \\
\hline Emotiva & 352.7 & 306.3 & 234.6 & $46.198(2)^{* *}$ & .20 \\
\hline Cognitiva & 343.0 & 306.5 & 245.1 & $32.077(2)^{* *}$ & .16 \\
\hline Empatia profesional & 378.8 & 307.9 & 203.9 & $100.805(2)^{* *}$ & .29 \\
\hline Emotiva & 360.9 & 300.5 & 232.0 & $54.527(2)^{* *}$ & .21 \\
\hline Cognitiva & 360.5 & 305.4 & 227.0 & $59.528(2) * *$ & .22 \\
\hline
\end{tabular}

Nota. $g l=$ grados de libertad; $* p<.05 ; * * p<.01$.

Tabla 10.

Comparación de los estilos parentales disfuncionales y la empatía personal y profesional, según los cursos de desarrollo personal, en estudiantes universitarios $(N=599)$

\begin{tabular}{|c|c|c|c|c|}
\hline \multirow[b]{2}{*}{ Variable y dimensiones } & \multicolumn{2}{|c|}{ Rango promedio } & \multirow[b]{2}{*}{$\begin{array}{c}\text { U de Mann- } \\
\text { Whitney }\end{array}$} & \multirow[b]{2}{*}{ PS } \\
\hline & $\begin{array}{c}\text { Con cursos } \\
(\mathrm{n}=192)\end{array}$ & $\begin{array}{l}\text { Sin cursos } \\
(\mathrm{n}=407)\end{array}$ & & \\
\hline Estilo disfuncional paterno & 279.68 & 300.55 & 33370.00 & - \\
\hline Indiferencia-negligencia & 287.43 & 284.58 & 35638.50 & - \\
\hline Autoritario-controlador & 275.41 & 309.34 & $31726.00 *$ & .44 \\
\hline Estilo disfuncional materno & 298.24 & 302.17 & 38908.00 & - \\
\hline Indiferencia-negligencia & 300.41 & 297.59 & 38463.00 & - \\
\hline Autoritario-controlador & 299.67 & 299.15 & 38608.50 & - \\
\hline Empatia personal & 325.09 & 246.82 & $28862.00 * *$ & .40 \\
\hline Emotiva & 319.15 & 259.41 & $31278.50 * *$ & .42 \\
\hline Cognitiva & 315.70 & 266.71 & $32681.00 * *$ & .37 \\
\hline Empatía profesional & 330.12 & 236.16 & $26814.50 * *$ & .39 \\
\hline Emotiva & 321.58 & 254.25 & $30287.50 * *$ & .38 \\
\hline Cognitiva & 322.64 & 252.00 & $29855.50 * *$ & .34 \\
\hline
\end{tabular}

Nota. $* * p<.01 ; * p<.05 ; \mathrm{PS}=$ probabilidad de superioridad. 
significativas, ya que se obtuvo un mayor puntaje en los estudiantes que sí llevaron cursos de desarrollo personal. Cabe mencionar que todas las diferencias significativas lograron un tamaño de efecto de nivel medio (Castillo, 2014).

\section{Discusión}

El presente estudio contó con una muestra 599 estudiantes universitarios provenientes de una universidad nacional y privada de Lima Metropolitana, Perú, con edades que fluctuaron entre $\operatorname{los} 21$ y 25 años $(M=23.20, \mathrm{DS}=3.34)$, en donde $65.9 \%$ eran mujeres y $34.1 \%$, hombres. Se recogió información de estudiantes de todos los años de estudio, siendo el sexto año el que agrupó un mayor número de estudiantes (54.6 \%).

A nivel descriptivo, se identificó que los estilos parentales disfuncionales en la subescala padre predominaron en un nivel medio en todas las carreras, sin embargo, se encontró un porcentaje ligeramente mayor de estudiantes de Medicina en el nivel alto de estilo disfuncional paterno. Asimismo, se encontró un patrón similar en los estilos parentales disfuncionales de la subescala madre, ya que los estudiantes alcanzaron un nivel medio en todas las carreras profesionales, aunque con un porcentaje ligeramente mayor de estudiantes de Medicina en el nivel alto de estilo disfuncional materno, lo cual indica que este grupo ha vivenciado un mayor nivel de estilos parentales disfuncionales durante su crianza que los alumnos de Psicología y Enfermería. Estos resultados guardan similitud con los hallazgos realizados por Peñaherrera y Moscoso (2016), en donde se identificó que los estudiantes de Medicina y Psicología presentaban con mayor recurrencia un funcionamiento familiar caótico y aglutinado, por lo que concluyen que la mayoría de los estudiantes de estas dos carreras pertenecían a familias disfuncionales.

Con respecto a la variable de empatía personal y profesional, los estudiantes de todas las carreras profesionales se ubicaron en un nivel medio de la escala, aunque hubo un mayor porcentaje de estudiantes de Enfermería con nivel alto de empatía personal. Esto último coincide con el estudio de Díaz et al. (2014), en donde se concluye que los estudiantes de Enfermería se caracterizan por tener niveles de empatía relativamente altos.

Por otra parte, Moreto et al. (2014) identificaron que el nivel de empatía los alumnos de Medicina podía cambiar conforme van avanzando en la formación profesional, sobre todo, de forma negativa, por lo cual señalan la importancia de intervenir en este proceso no solo enseñando cosas nuevas, sino previniendo su pérdida. Esto coincide con los mayores niveles de empatía encontrados en los estudiantes de 21 y 22 años del presente estudio.
Siguiendo con la variable empatía, se encontró un mayor porcentaje de estudiantes de Psicología con un nivel bajo de empatía personal, dato que llama la atención debido a que la empatía es un requisito indispensable para el ejercicio profesional del psicólogo. Los cursos de la carrera de Psicología deben promover el desarrollo de la empatía, la misma que es de suma importancia en el vínculo terapeuta-paciente. $\mathrm{Al}$ respecto, múltiples estudios relacionan una mejor empatía con una mejor competencia clínica, así como con una mejor comunicación y relación médico-paciente; en concreto, Ogle, Rubin y Siegler (2013) refieren que altos niveles de empatía se relacionan con mayor facilidad para expresar síntomas y preocupaciones por parte de los pacientes, con lo que no solo se obtiene una mejor anamnesis y precisión diagnóstica, sino también una mayor participación del paciente, mejor educación en salud, mejor calidad de vida en general del paciente y una importante reducción del estrés.

Por otra parte, en el análisis correlacional se hace evidente la presencia de correlaciones inversas, aunque en un nivel bajo, en las dimensiones empatía personal cognitiva y empatía profesional cognitiva con respecto al estilo parental disfuncional autoritario-controlador, así como una mayor empatía personal emotiva en relación con un menor estilo disfuncional paterno de indiferencia-negligencia. Frente a estos resultados, se puede deducir que los estilos disfuncionales paternos inciden, en parte, sobre el desarrollo de la empatía.

Al respecto, es claro que la multicausalidad del desarrollo de la empatía se encuentra inmersa en un clima familiar y su respectiva funcionalidad, ya que se considera a la familia y su sistematización como el medio donde se aprende a serempático. Esto guarda relación con el estudio de Madera et al.(2015), en donde se concluye que los niveles de empatía pueden variar dependiendo del sexo, la funcionalidad familiar y el rendimiento académico, por lo cual se propone la implementación de estrategias pedagógicas en las áreas humanas que involucren a las familias de los estudiantes con el fin de mejorar, de este modo, los niveles de empatía y la atención en salud.

Adicionalmente, se encontraron asociaciones directas entre las dimensiones empatía personal emotiva y empatía profesional emotiva con el estilo disfuncional materno autoritario-controlador, por lo que podría suponerse que hay un mayor desarrollo de la empatía emotiva y profesional frente a una percepción elevada en los estilos disfuncionales relacionados a la figura materna como autoritaria-controladora. Este dato es relevante debido a que se puede evidenciar la importancia de las variables culturales específicas, en donde la figura femenina se ve como la proveedora del cuidado y la crianza. De esta manera, la presencia de factores culturales puede estar interfiriendo en los resultados, de modo que ampliar su estudio en futuras investigaciones facilitaría la 
110

identificación de los factores específicos que intervienen en el desarrollo de la empatía.

Por otra parte, a nivel comparativo se encontró que, según el género, las diferencias fueron significativas en la variable empatía personal y su dimensión emotiva, ya que en ambas hubo un mejor nivel de empatía en las mujeres. Esto guarda relación con el estudio realizado por Esquerda et al (2015), en donde se encontró que quienes presentan mayor empatía son las estudiantes de género femenino.

Asimismo, según la carrera profesional, se hallaron diferencias significativas en las dimensiones autoritariocontrolador e indiferencia-negligencia del estilo disfuncional paterno, ya que en ambas dimensiones los estudiantes de Medicina obtuvieron un mayor puntaje. Considerando que los estilos parentales disfuncionales guardan relación con diversas patologías mentales, se podría deducir que los estudiantes de Medicina presentan mayor vulnerabilidad o factores de riesgo que podrían explicar sus niveles de empatía y la pérdida de esta conforme avanzan en la formación profesional, tal como se encontró en el presente estudio. A partir de esto, se hace hincapié en que se debe dar un entrenamiento en empatía en la formación médica, ya que, si un médico es capaz de empatizar correctamente con el enfermo, este último manifestará mayor satisfacción, adherencia al tratamiento y voluntad para entregar información sensible con el fin de establecer su diagnóstico. Esto concuerda con lo encontrado por Donoso (2014), donde se afirma que la empatía aumenta la participación y la educación del paciente, al igual que permite disminuir el malestar emocional, y, por tanto, incrementar su calidad de vida.

Además de esto, se encontraron diferencias significativas en la empatía personal y profesional de los estudiantes de Enfermería, ya que demostraron mayores puntajes que los encontrados en los estudiantes de otras carreras. Al respecto, se pueden considerar algunas variables en la labor y formación de los enfermeros que influyen sobre este resultado, ya que, como reportan Díaz et al. (2014), algunos de los motivos por los cuales los estudiantes de Enfermería evidencian un alto nivel de empatía refieren a la toma de perspectiva, el cuidado con compasión y ponerse en los zapatos del paciente; factores posiblemente asociados a un alto nivel de motivación y compromiso de los estudiantes para prestar la atención al dolor humano y cuidado de los pacientes, en donde la empatía sería vista como una precursora del cuidado genuino. En el presente estudio, de las tres profesiones evaluadas, la carrera de Enfermería es la que en su labor práctica se encuentra con mayor contacto con los pacientes tanto en lo físico como en lo emocional.

Adicionalmente, los resultados a partir de si los estudiantes llevaron a cabo o no cursos de desarrollo personal en la carrera profesional apuntan a que las variables empatía personal y profesional y sus dimensiones también logran diferencias significativas, debido a un mayor puntaje en los estudiantes que síllevaron cursos de desarrollo personal. Este resultado indica que la formación profesional del personal de salud incide no solo en la adquisición de aprendizaje y habilidad técnica, sino también en la formación personal, como en lo relacionado al desarrollo y mantenimiento de la empatía, lo cual termina brindando a la sociedad profesionales que humanizan la salud.

Cabe mencionar que, habitualmente, se habla de simpatía y empatía como sinónimos, pero existen claras diferencias conceptuales entre ellas. Al respecto, García y Alarcos (2002) explican que la simpatía ocurre cuando una persona, queriendo comprender a otra, experimenta sus mismas emociones, y que el objetivo de la empatía no es lograr experimentar los sentimientos ajenos, sino captarlos de la manera más justa posible a como son vividos. Por consiguiente, la empatía no solo incluiría elementos afectivos, sino también cognitivos y conductuales, de modo que esta puede ser enseñada y ejercitada para ser transformada en un hábito propio del profesional de la salud (García \& Alarcos, 2002).

Finalmente, es importante señalar que los resultados expuestos muestran coeficientes de correlación bajos pese a haber un tamaño considerable de muestra, por lo cual es recomendable tomar lo resultados expuestos con prudencia. Se propone realizar futuras investigaciones que contrasten sus resultados a los reportados en el presente estudio.

Por todo lo anterior, se puede concluir que el estudio reporta la ausencia de correlación entre la variable estilos parentales disfuncionales (paterno y materno) con la variable empatía. Sin embargo, se reportan correlaciones inversas -pero de magnitud baja- entre las dimensiones empatía personal cognitiva y empatía profesional cognitiva en relación con el estilo parental disfuncional autoritario-controlador del padre; así como asociaciones directas entre el estilo disfuncional materno autoritario-controlador y las dimensiones empatía personal emotiva y empatía profesional emotiva; al igual que una relación positiva entre los estudiantes que llevaron cursos de desarrollo personal y los puntajes altos en las variables empatía personal y profesional. Sin embargo, estos resultados deben ser tomados con precaución ante los pequeños tamaños del efecto reportados, por lo que los hallazgos expuestos quedan abiertos a la discusión y podrán ser parte de revisiones posteriores.

\section{Referencias}

Baumrind, D. (1996). The discipline controversy revised. Family Relations, 45(4), 405-414. Recuperado de https://www.jstor. org/stable/pdf/585170.pdf?seq=1\#page_scan_tab_contents 
Bilbao, J., Iglesias, J., Díaz, V., Crespo, E., Marina, L., \& Alcocer, A. (2015). Orientación empática en estudiantes de medicina de las Universidades Libre y San Martín, Barranquilla, Colombia. Revista de la facultad de medicina, 63(4), 657663. doi: http://doi.org/10.15446/revfacmed.v63.n4.49715

Camacho, J. (2006). Aproximación a la empatía en la relación médico-paciente. Revista Bioética y Debate, 43(2), 8-12. Recuperado de https://dialnet.unirioja.es/servlet/ articulo? codigo $=1984600$

Carrasco, D., Fasce, E., \& Pérez, C. (2014). Propiedades psicométricas de la Escala de Empatía Personal y Profesional de Yarascavitch en estudiantes de odontología chilenos. Revista de Educación en Ciencias de la Salud, 11(2), 147-153. Recuperado de https://dialnet.unirioja.es/servlet/ articulo? codigo $=6287573$

Castillo, R. (2014). Reporte del tamaño del efecto en los artículos de tres revistas de psicología peruanas en los años 2008 al 2012 (Tesis de licenciatura). Universidad Nacional Mayor de San Marcos, Lima-Perú.

Cuba, M., Jurado, A., \& Estrella, E. (2011). Evaluación del cumplimiento de los atributos de la Atención Primaria y grado de satisfacción de los usuarios de un establecimiento de primer nivel de atención. Revista Médica de Cayetano Heredia, 22(1), 4-9. Recuperado de http://www.scielo.org.pe/scielo. php?script=sci_arttext\&pid=S1018-130X2011000100002

Davis, M. H. (1994). Empathy: a social psychological approach. New York: Brown \& Benchmark.

Díaz, V., Muñoz, G., Duarte, N., Reyes, M., Elvira, S., Calzadilla, A., \& Alonso, L. (2014). Empatía en estudiantes de enfermería de la Universidad Mayor, sede Temuco, IX región, Chile. Aquichan, 14(3), 388-402. doi: dx.doi. org/10.5294/aqui.2014.14.3.9

Díaz, V., Salas, D., Bracho, F., \& Ocaranza, J. (2015). Empatía en estudiantes de kinesiología. Universidad Mayor, sede Temuco, Chile. Revista Ciencia Salud, 12(3), 383-393. doi: https://dx.doi.org/10.12804/revsalud13.03.2015.05

Donoso, C. (2014). La empatía en la relación médico-paciente como manifestación del respeto por la dignidad de la persona. Una aportación de Edith Stein. Persona y Bioética, 18(2), 184-193. doi: https://dx.doi.org/10.5294/pebi.2014.18.2.8

Esquerda, M., Yuguero, O., Viñas, J., \& Pifarré, J. (2015). La empatía médica, ¿nace o se hace? Evolución de la empatía en estudiantes de medicina. Atención Primaria, 48(1), 8-14. doi: https://dx.doi.org/10.1016/j.aprim.2014.12.012

García, J., \& Alarcos, F. (2002). 10 palabras claves en humanizar la salud. Madrid: Casa del Libro.

García, R., \& Gálvez, N. (2016). Calidad de atención asociada a la satisfacción del usuario externo en los establecimientos asistenciales de salud: Minsa-Essalud, Chongoyape, Perú. Revista Tzhoecoen, 8(2). Recuperado de http://revistas.uss. edu.pe/index.php/tzh/article/view/383/371

Hernández, R., Fernández, C., \& Baptista, M. (2016). Metodología de la investigación (5. ${ }^{\mathrm{a}}$ ed.). México: McGRAW-HILL.
Lain, P. (1964). Relación médico enfermo: historia y teoría. Madrid: Ediciones Castilla.

Madera, M., Tirado, L., \& González, F. (2015). Factores relacionados con la empatía en estudiantes de medicina de la Universidad de Cartagena. Revista Clínica de Medicina de Familia, 18(3), 185-192. doi: https://dx.doi.org/10.4321/ S1699-695X2015000300003

Manterola C., \& Otzen, T. (2017). Los sesgos en Investigación Clínica. International Journal of Morphology, 33(3), 1156-1164. doi: https://dx.doi.org/10.4067/ S0717-95022015000300056

Matalinares, M., Raymundo, O., \& Baca, R. (2014). Propiedades psicométricas de la Escala de Estilos Parentales (MOPS). Revista Persona, 17(1), 95-121. doi: https://dx.doi. org/10.26439/persona2014.n017.291

Moreto, G., González, P., Pessini, L., \& Craice, M. (2014). La erosión de la empatía en estudiantes de Medicina: reporte de un estudio realizado en una Universidad de Sao Paulo, Brasil. Atención Familiar, 21(1), 16-19. doi: http://doi. org/10.1016/S1405-8871(16)30005-0

Ogle, C., Rubin, D., \& Siegler, I. (2013). The impact of the developmental timing of trauma exposure on PTSD symptoms and psychosocial functioning among older adults. Developmental Psychology, 49(11), 2191-2200. doi: http:// doi.org/ 10.1037/a0031985

Otzen, T., \& Manterola C. (2017). Técnicas de muestreo sobre una población a estudio. International Journal of Morphology, 35(1), 227-232. doi: https://dx.doi. org/10.4067/S0717-95022017000100037

Parker, G., Tupling, H., \& Brown, L. (1979). A parental bonding instruments. British Journal of Medical Psychology, 37(52), 1-10. doi: http://doi.org/10.1111/j.2044-8341.1979. tb02487.x

Peñaherrera, J., \& Moscoso, D. (2016). Personalidad y funcionamiento familiar en estudiantes de medicina y psicología de la universidad del Azuay. Recuperado de http://dspace. uazuay.edu.ec/bitstream/datos/6355/1/12523.pdf

Salcedo-Rioja, M., \& Díaz-Narváez, V. (2015). Empatía en estudiantes de odontología de la Universidad Nacional Mayor de San Marcos (Perú). Salud Uninorte, 31(3), 565-574. doi: https://dx.doi.org/10.14482/sun.31.3.6801

Sánchez, H., \& Reyes, C. (2015). Metodología y diseños en la investigación científica (5. ${ }^{\text {a }}$ ed.). Lima: Editorial Visión Universitaria.

Sánchez, J. (2010). Estudio comparativo de empatía médica, sensibilidad emocional y características psicosociodemográficas entre estudiantes de medicina y médicos especialistas docentes en Venezuela. Recuperado de https://repo sitorio.uam.es/bitstream/handle/10486/4764/31383_san chez_delgado_jose.pdf? sequence $=1$

Yarasckavitch, C., Regerhr, G., Hodges, B., \& Haas, D. (2009). Changes in dental student empathy during training. Journal of Dental Education, 73(4), 509-517. Recuperado de https:// www.ncbi.nlm.nih.gov/pubmed/19339438 